\title{
High-throughput roll-to-roll X-ray characterization of polymer solar cell active layers
}

\author{
Böttiger, Arvid P.L.; Jørgensen, Mikkel; Menzel, Andreas; Krebs, Frederik C; Andreasen, Jens Wenzel
}

Published in:

Journal of Materials Chemistry

Link to article, DOI:

10.1039/c2jm34596j

Publication date:

2012

Document Version

Publisher's PDF, also known as Version of record

Link back to DTU Orbit

Citation (APA):

Böttiger, A. P. L., Jørgensen, M., Menzel, A., Krebs, F. C., \& Andreasen, J. W. (2012). High-throughput roll-toroll X-ray characterization of polymer solar cell active layers. Journal of Materials Chemistry, 22(42), 2250122509. https://doi.org/10.1039/c2jm34596j

\section{General rights}

Copyright and moral rights for the publications made accessible in the public portal are retained by the authors and/or other copyright owners and it is a condition of accessing publications that users recognise and abide by the legal requirements associated with these rights.

- Users may download and print one copy of any publication from the public portal for the purpose of private study or research.

- You may not further distribute the material or use it for any profit-making activity or commercial gain

- You may freely distribute the URL identifying the publication in the public portal 


\title{
Materials Chemistry
}

Cite this: J. Mater. Chem., 2012, 22, 22501

www.rsc.org/materials

PAPER

\section{High-throughput roll-to-roll X-ray characterization of polymer solar cell active layers $\dagger$}

\author{
Arvid P. L. Böttiger, ${ }^{a}$ Mikkel Jørgensen, ${ }^{a}$ Andreas Menzel, ${ }^{b}$ Frederik C. Krebs ${ }^{a}$ and Jens W. Andreasen ${ }^{\star a}$
}

Received 13th July 2012, Accepted 7th September 2012

DOI: $10.1039 / \mathrm{c} 2 \mathrm{jm} 34596 \mathrm{j}$

Synchrotron-based X-rays were used to probe active materials for polymer solar cells on flexible polyester foil. The active material was coated onto a flexible 130 micron thick polyester foil using rollto-roll differentially pumped slot-die coating and presented variation in composition, thickness, and additives. The coated foil was passed through a synchrotron X-ray beam on a small unit comprising unwinder and winder for the foil, an X-ray probe station, and a barcode reader for sample registration. Foil lengths of 10 meters were probed and yielded X-ray scattering data for approximately every $1 \mathrm{~cm}$, probing linear variations in processing and coating parameters along the foil. The demonstration shows that real-time structural characterization of roll-to-roll coating at realistic web-speeds is feasible using synchrotron radiation. Off-line characterization with lower spatial resolution would be possible with dedicated laboratory instruments. We found that poly(3-hexyl)thiophene (P3HT) only crystallizes at a ratio above $1: 2$ with phenyl-C61-butyric acid methyl ester (PCBM) and that an optimum addition of 2 vol\% chloronaphthalene $(\mathrm{CN})$ as a processing additive significantly improves polymer crystallinity and crystallite size. In coated films thinner than $275 \mathrm{~nm}$, textured poly(3-hexyl)thiophene crystallites with the lamellar stack aligned with the substrate dominate, similar to what is observed for spin-coated films.

\section{Introduction}

Some of the defining properties of polymer solar cells are characterized by the tremendous ease and speed with which they can be processed. The typical polymer solar cell is comprised of a multilayer stack of organic and inorganic materials that are processed one after the other using solution-based roll-to-roll coating and printing. For each layer the morphology and interface behavior present an intricate and complex dependency on the processing conditions and parameters. When polymer solar cell films are prepared by spin coating in a batch process, subsequent characterization is comparable in terms of the time required for the preparation, but when each layer is applied in a continuous process, roll-to-roll (R2R), new challenges must be met: the amount of data that needs to be efficiently collected and analyzed to characterize a given process or materials combination scales with the coated area. For the typical R2R coated

${ }^{a}$ Department of Energy Conversion and Storage, Technical University of Denmark, Frederiksborgvej 399, DK-4000 Roskilde, Denmark. E-mail: jewa@dtu.dk

${ }^{b}$ Paul Scherrer Institut, 5232 Villigen PSI, Switzerland

$\uparrow$ Electronic supplementary information (ESI) available: Quantification of the P3HT:PCBM ratio in the ratio gradient experiment by UV-vis characterization of the R2R printed modules, an example of a 100 reflection peak profile with Gaussian and Lorentzian fits, distribution of extracted parameters from film with unvaried composition, variation of 100 reflection azimuthal spread with gradient, an example of GIWAXS data as recorded, and animations showing the raw SAXS data acquired are available online. See DOI: 10.1039/c2jm34596j polymer solar cell the web speeds employed are in the range of $1-10 \mathrm{~m} \mathrm{~min}^{-1}$ using web widths of $305 \mathrm{~mm}$. This implies that an enormous amount of area is coated in a given amount of time compared to the speed at which that same area can be characterized using conventional methods such as optical methods, scanning probe techniques, discrete electrical measurements, and $\mathrm{X}$-ray scattering. Unless the analysis method has the same throughput as the processing methods it is unlikely to be useful as a technique to optimize a given process or materials system. The characterization methods that have been demonstrated in an R2R setting are I-V characterization (current-voltage), optical microscopy, UV-visible spectroscopy, light beam induced current (LBIC), and incident photon to electron conversion efficiency (IPCE). ${ }^{\mathbf{1} 2}$ In addition to those techniques, ellipsometry, dark lock-in thermography, electroluminescence imaging, and optical coherence tomography have the potential to become $\mathrm{R} 2 \mathrm{R}$ compatible. ${ }^{3-6}$ Common to all these methods is that they yield macroscopic measures of the polymer solar cell such as structural and functional variations of the solar cells with a resolution of around 100 micron and particle detection down to around 10 micron. If one wishes to explore the microscopic nature of the organic solar cell stack on the micro- or nanoscale, new and rapid characterization tools are needed. One of the most powerful methods to probe the morphology and molecular organization of active materials in polymer solar cells is X-ray scattering methods. Intense synchrotron X-ray light sources allow X-ray characterization to be carried out at speeds compatible with roll-to-roll processing. There are a number of 
challenges that must be overcome, among which are an efficient sample geometry on flexible plastic substrates and an automated sampling method coupled to an automated data analysis protocol. Until now very little work has been dedicated to study $\mathrm{R} 2 \mathrm{R}$ processing using X-rays. Of relevance, though, are the recent intensive studies by Schmidt-Hansberg et al. of the drying process of polymer solar cell active layer solutions, in a knifecoating setup ("doctor-blading”). ${ }^{7-9}$ In these studies, X-ray diffraction of the drying film is measured in grazing incidence, while simultaneously monitoring the film thickness with a visiblelight reflectometer. It was found for bulk heterojunctions of poly(3-hexyl)thiophene and [6,6]-phenyl-C61-butyric acid methyl ester (P3HT/PCBM) that the nanomorphology, phase separation, and crystallinity could be controlled by drying temperature through reduced molecular kinetics. ${ }^{7}$ The effect of the P3HT:PCBM blend ratio on structural evolution of a drying doctor-bladed film indicated a strong molecular interaction between the two compounds, inhibiting P3HT $\pi-\pi$ packing at a large excess of $\mathrm{PCBM}^{8}$ At an excess of P3HT it was found that crystallization of PCBM is inhibited long past the solubility limit. The addition of a "poor solvent" for P3HT (cyclohexane) caused aggregation of $\mathrm{P} 3 \mathrm{HT}$ in solution and consequently a broader orientation distribution of crystallites as compared to the substrate/surface-induced crystallization from pure chlorobenzene. ${ }^{9}$ While clearly relevant for the understanding of coating of polymer solar cell active layers these studies are essentially batch experiments and, therefore, still limited with respect to R2R coating. For efficient exploration of the materials and processing parameter space, a truly $\mathrm{R} 2 \mathrm{R}$ compatible implementation is required. In this work we demonstrate roll-to-roll characterization of active layers for polymer solar cells using synchrotron Xray scattering by employing a simple unwinder-registrationwinder unit and flexible plastic foil as the substrate for the measurements, as shown in Fig. 1. Three different gradient experiments show the possibilities for exploring the structural phase diagram of the multicomponent parameter space of polymer solar cell processing: donor/acceptor ratio, active layer thickness, and concentration of a solvent additive.

\section{Results and discussion}

With thousands of measurements per experiment and diffraction data from area detectors, efficient analysis methods are required to evaluate the results. From inspection of the raw data we identified and extracted the following parameters from the data recorded on the Megapixel SAXS detector: integrated intensity, width, and position of the 100 reflection of P3HT, both from the peak centered on the surface normal corresponding to textured P3HT aligned with the substrate and for a segment of the diffraction ring corresponding to randomly oriented P3HT crystallites (Fig. 2). To observe the 100 reflection corresponding to textured $\mathrm{P} 3 \mathrm{HT}$, a relatively high $\mathrm{X}$-ray incidence angle of $1.5^{\circ}$ was required (see also the Experimental section) with the beam thus penetrating into the PET substrate causing strong background scattering in the WAXS detector. Combined with air scattering, this prevented the observation of diffraction signals from either P3HT or PCBM in the GIWAXS data recorded on the WAXS detector, and consequently, these data were not analyzed further. Thus, in the following, only data from the
SAXS detector are discussed. For less crystalline polymers, it would likely be advantageous to choose a more shallow incidence angle to optimize the signal to noise ratio for GISAXS.

The parameters related to the P3HT 100 reflections were extracted by fitting a Gaussian function to the integrated peaks after subtracting a linear background function, yielding information about the amount of crystalline P3HT from the area of the fitted Gaussian, the crystalline coherence length from the fitted peak width through the Scherrer equation, and the lamellar packing density of the crystalline structure from the peak position. Although it is generally agreed that size-strain analysis on a single reflection cannot be used in a quantitative manner, we examined the possibility of separating peak broadening effects of size and strain by fitting a pseudoVoigt peak function where the GaussLorentz mixing parameter might reveal useful information on trends in size/strain. We found, however, that the broad peaks are equally well fitted by "Gaussian" and "Lorentzian" pseudoVoigt functions therefore yielding very poorly determined mixing factors when fitted freely (see ESI Fig. S3†), and thus resorted to purely Gaussian fits to extract the parameters described above. The term coherence length is used to emphasize that these figures merely describe the limits to coherently diffracting regions without implying definite information on defects, strain or crystallite size.

In all the experiments, crystallites with the lamellar stack aligned with the substrate clearly dominate, similar to the texture observed for spin coated P3HT. ${ }^{10}$ Because of difficulties in obtaining an automated, stable fit to the generally weak signal from untextured crystallites, the coherence lengths for these were found to be too unreliable and were not analyzed further, and only intensities and $d$-spacings were extracted. In some cases, clear outliers can be identified, caused by tilting of the substrate foil when moving to the next sampling positions. These are obvious, however, as lying many standard deviations from the mean, and represent less than $5 \%$ of the data points. The effect of sample position instability is most severe with respect to the scattering intensity, and much less so with respect to reflection peak widths and position. The maximal theoretical bias of the 100 peak position corresponds to scattering from either side of the film width of $10 \mathrm{~mm}$ equaling $0.08 \AA$ in $d$-spacing. In general, standard deviations half of this or less are observed, except for very weak peaks. For constant composition, we observe relative standard deviations of $0.1 \%$ for the $100 \mathrm{~d}$-spacing of textured $\mathrm{P} 3 \mathrm{HT}, 20.7 \%$ for the integrated intensity and 3.2\% for the coherence length (see ESI Fig. S4-S6†).

The data analysis was performed post-acquisition, but we consider it feasible to apply these analysis methods online, to allow monitoring of meaningful physical parameters during R2R coating. Thanks to the large amount of sampling points, we get information about correlations between these properties and their correlation with the controlled parameter with very high statistical significance.

A small-angle scattering pattern along the substrate surface (i.e. along $q_{x y}$ ) was extracted by integrating the scattered intensity in a region around a fixed $q_{z}$ corresponding to the position of the Yoneda peak (Fig. 2). For each point we integrated data from four $100 \mathrm{~ms}$ exposures, but sufficient intensity is acquired in a single exposure. This allows for measurements with continuous foil movement at speeds of up to $6 \mathrm{~m} \mathrm{~min}^{-1}$ with the same spatial resolution as in the present experiments, thus enabling real-time 


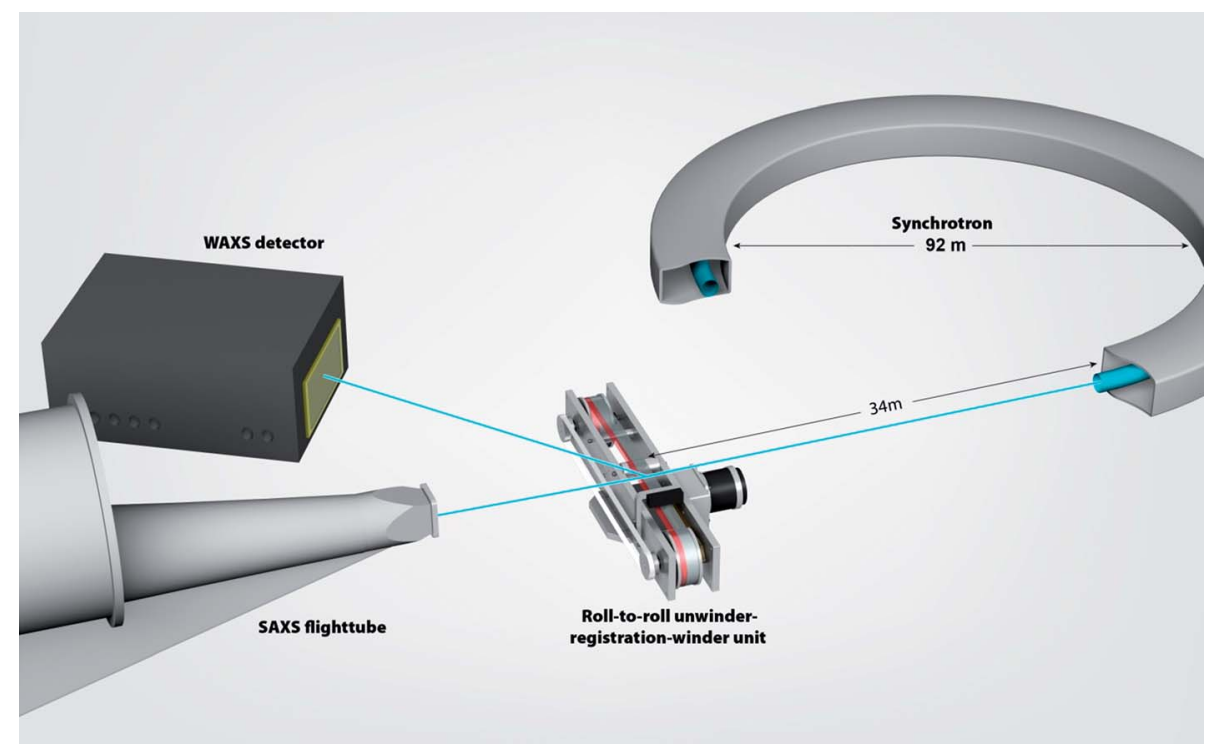

Fig. 1 Artist's rendition of the experiment showing the X-ray source (the synchrotron), the roller unit, and the detectors (WAXS detector and SAXS flight tube entry). Note that the WAXS data were found not to provide usable data in the reported experiments (see explanation below).

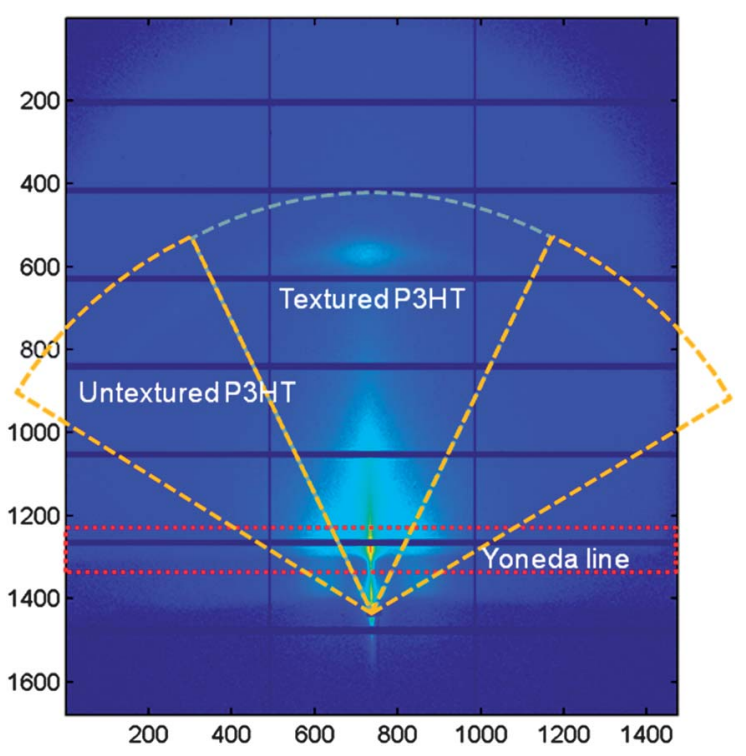

Fig. 2 A SAXS pattern from an R2R experiment showing the integration regions used to extract structural parameters of the film. The central region, covering an azimuthal angle of $50^{\circ}$, was chosen to represent the scattering of P3HT crystallites with the lamellar stack aligned with the substrate ("textured P3HT"). The two regions to the side, outlined in yellow, were used to integrate scattering from P3HT crystallites with random orientation ("untextured $\mathrm{P} 3 \mathrm{HT}$ "). The rectangular region, outlined in red, corresponds to the region integrated to extract the GISAXS pattern along the "Yoneda line". ${ }^{12}$ The numbering indicates the pixels. For an example of an integrated peak profile of the textured P3HT reflection, please refer to the ESI. $\dagger$

analysis of coating processes. An optimized laboratory instrument $^{11}$ can deliver $10^{7}-10^{8}$ photons on the sample i.e. 4-5 magnitudes lower flux as compared to an insertion device synchrotron beam line. The higher divergence of the laboratory instrument means that more crystallites are in diffraction condition for polycrystalline films at a given incidence angle. Accepting a lower spatial resolution (e.g. an exposure every $5 \mathrm{~cm}$ ), gradient experiments as the ones reported here could be completed in a few days with an exposure time of $1000 \mathrm{~s}$.

The GISAXS patterns integrated along the Yoneda line ${ }^{12}$ are very susceptible to variations in the sample position, and contain a substantial scattering contribution from the substrate because of the high incidence angle. We have consequently only evaluated large changes in scattering intensity, as was observed in the ratio gradient experiment (discussed below).

\section{Thickness gradient}

The parameters for the textured and untextured P3HT 100 peaks are shown as a function of nominal dry film thickness in Fig. 3. A smoothed line to guide the eye is added to each dataset by calculating a weighted linear least squares local regression using a $2^{\text {nd }}$ degree polynomial model across a data span of $15 \%$ of the total number of data points. Outliers outside six mean absolute deviations are assigned zero weight. The lighter colored band shows the standard deviation of the data points in the smoothing span centered on the corresponding data point, with respect to the smoothed mean. Some outliers representing very poor fits to the data are outside the plot region, but increase the standard deviation significantly (e.g. $d$-spacings of untextured crystallites in thin films, lower panel of Fig. 3).

The scatter in the data, caused by sample movement and film inhomogeneity, does not hide the clear trends, showing that the amount of crystalline textured P3HT increases with film thickness, but with a distinct maximum at $272 \mathrm{~nm}$ (top panel). With a larger thickness, untextured crystallites appear. The textured crystallite coherence length shows a broad maximum for a slightly thinner film, at $246 \mathrm{~nm}$ (Fig. 3, middle panel). For both textured and untextured crystallites, the lamellar spacing decreases slightly for the thickest films but with a maximum at $240 \mathrm{~nm}$ for the textured crystallites (lower panel).

In the scatterplots (Fig. 4) we observe a positive correlation between the lamellar $d$-spacing and the coherence length (top 


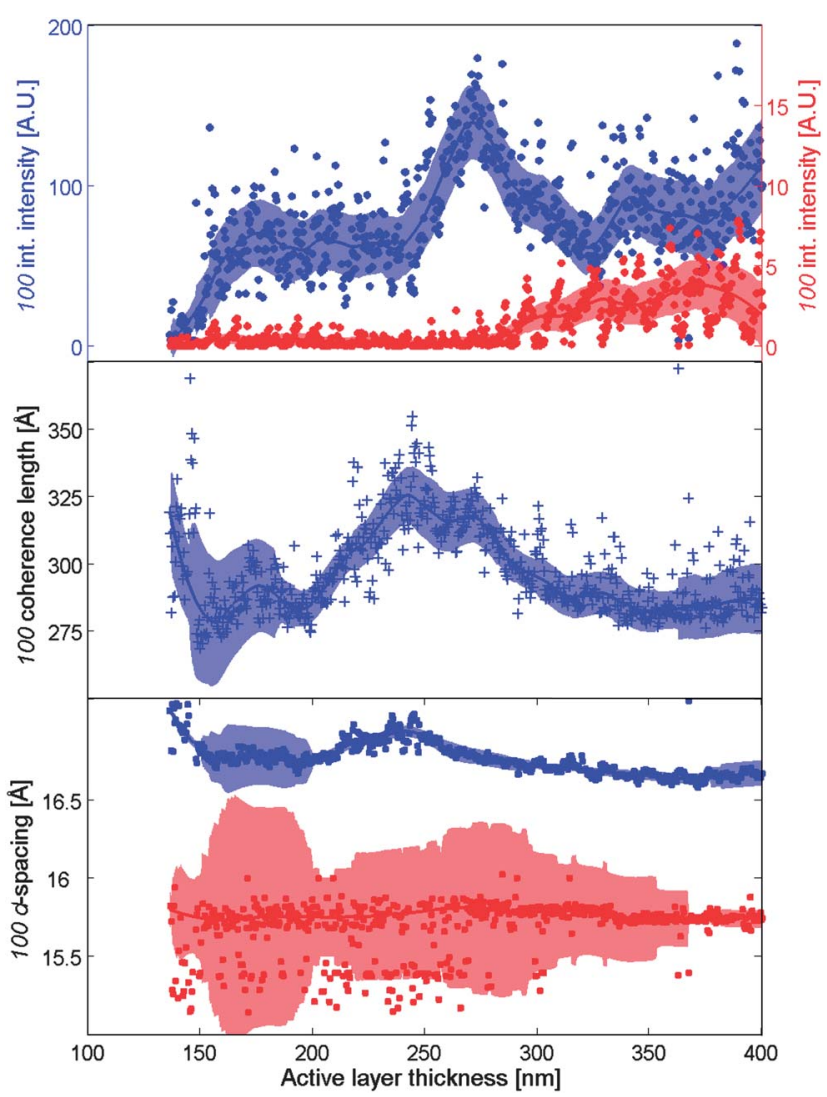

Fig. 3 Parameters of the crystalline P3HT 100 reflections shown as a function of the controlled gradient parameter, thickness. Data for the textured crystallites are shown in blue, for the untextured crystallites in red. Coherence lengths for untextured crystallites are not shown. A smoothed line is shown to guide the eye, with the associated band of one standard deviation of the data points with respect to the smoothed mean (see also main text for further explanation).

panel), but no correlation with the scattering intensity (middle panel). There is a positive correlation between integrated intensity and coherence length (lower panel). This is to be expected for a reflection from a population of a textured, semi-crystalline polymer, as in this case, both coherence length and integrated intensity will be related to the crystallinity of the polymer. The points corresponding to the smallest integrated intensity (below $\sim 15$, mostly dark blue) represent measurements where the 100 peak was ill defined, because the sample foil tilted during movement to a new measuring position. The recorded values in these points are thus viewed to be less accurate.

To summarize, we observe that, (1) up to $272 \mathrm{~nm}$, the crystalline $\mathrm{P} 3 \mathrm{HT}$ component of the active layer film is completely dominated by the textured population of crystallites presumably formed from nuclei in contact with the substrate, with the amount increasing with film thickness. (2) The largest of these crystallites occurs in films of 225-275 nm thickness. (3) The thickest films with longer drying times allow better organization of the polymer chains, and thus a shorter lamellar stacking, although the largest crystallites have the largest lamellar spacing, presumably due to steric hindrance during crystallization. (4) In films thicker than $275 \mathrm{~nm}$, kinetics favor the formation of new crystalline nuclei in the drying film, with random orientation, and (5) these untextured crystallites adopt a different structure polymorph than the textured crystallites, presumably with partly interdigitated side chains as evidenced by very short lamellar spacings (form II identified by Prosa et $a l .{ }^{13}$ ), or, the monoclinic structure identified by Brinkmann et al. ${ }^{14}$ also characterized by a short lamellar packing distance.

\section{Ratio gradient}

The 100 peaks, corresponding to both textured and untextured crystallites, first appear at a ratio of $1: 2$ between P3HT and PCBM (Fig. 5). At first, the scattering intensity passes through a local maximum at about $40 \%$ P3HT and again increases for the highest P3HT:PCBM ratios above 1:1. The 100 coherence length of the textured crystallites shows a broad minimum at $1: 1$ ratio, whereas the lamellar $d$-spacing decreases with $\mathrm{P} 3 \mathrm{HT}$ concentration, through a local minimum at $\sim 42 \mathrm{wt} \%$ P3HT. The lamellar $d$-spacing of the untextured crystallites is constant at the very short distance of $15.7 \AA$ in corroboration of the results obtained in the thickness gradient experiment.

Scatterplots show that the coherence length and lamellar $d$-spacing are uncorrelated (but the largest coherence lengths seen in the thickness gradient experiment are not observed here), whereas there is a negative correlation between integrated intensity and $d$-spacing (Fig. 6, top and middle panels). There is a

\section{thickness}
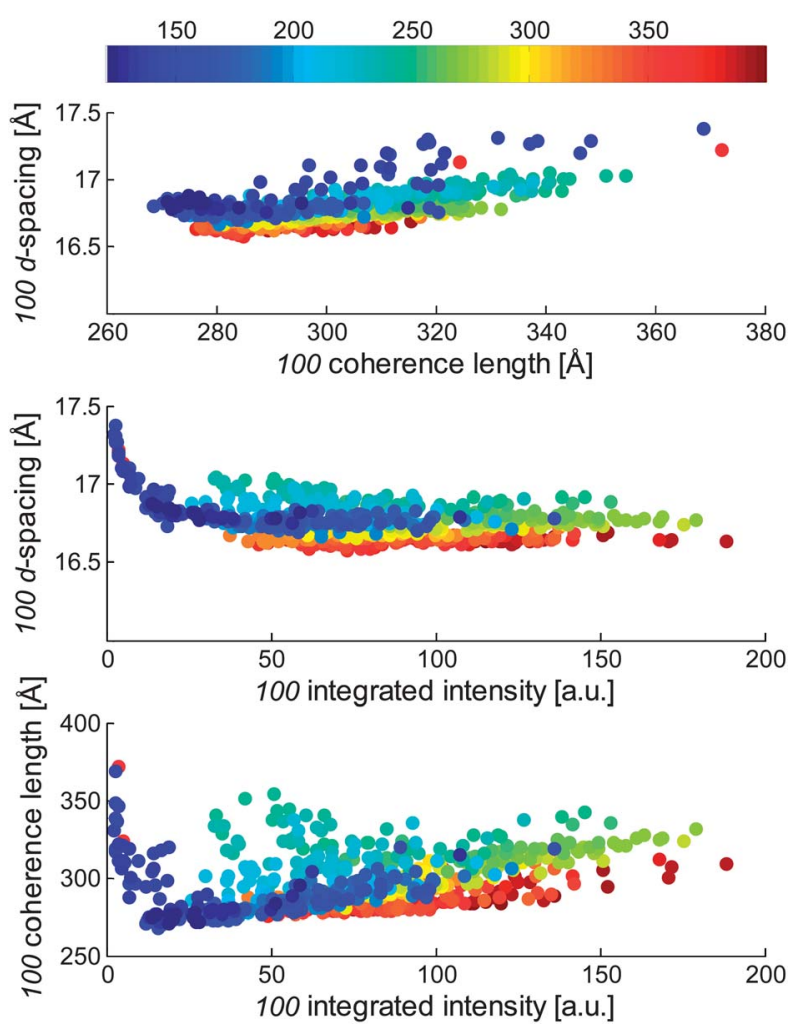

Fig. 4 Scatterplots showing relations between the parameters extracted from P3HT 100 reflections of textured crystallites for the thickness gradient experiment. Top panel: crystallite coherence length $v s . d$ spacing. Middle panel: integrated intensity $v s$. $d$-spacing. Lower panel: integrated intensity $v s$. crystallite coherence length. The color grading corresponds to the nominal film thickness, indexed as shown on the color bar at the top of the figure. 


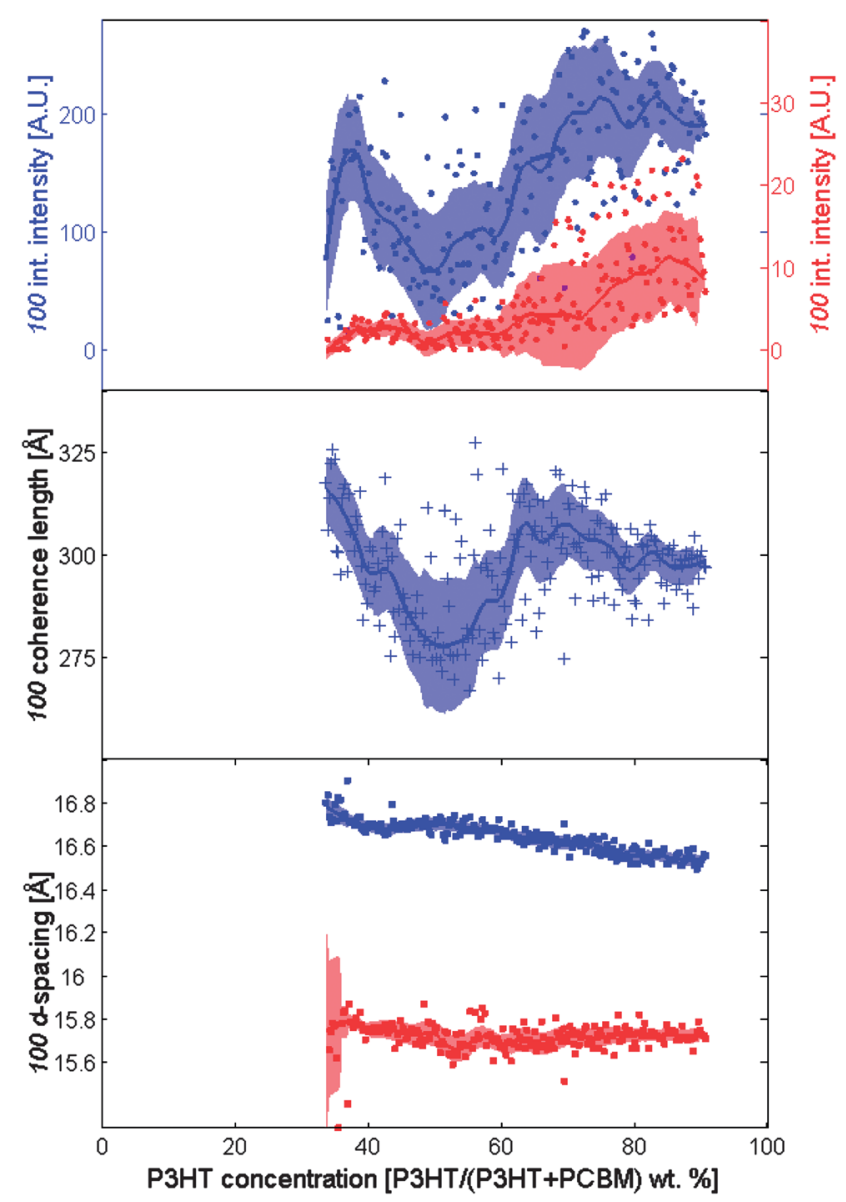

Fig. 5 Parameters of the crystalline P3HT 100 reflections shown as a function of the controlled gradient parameter, P3HT concentration $[\mathrm{P} 3 \mathrm{HT} /(\mathrm{P} 3 \mathrm{HT}+\mathrm{PCBM})$ by weight $]$. The data for the textured crystallites are shown in blue, for the untextured crystallites in red. Coherence lengths for untextured crystallites are not shown. No data are available below $37 \%$ P3HT where there is no P3HT diffraction signal.

clear positive correlation between integrated intensity and the 100 coherence length (Fig. 6, lower panel).

The GISAXS patterns show a very dramatic change at exactly the point where P3HT crystallites appear, at the ratio of $1: 2$ P3HT:PCBM (Fig. 7).

The GISAXS intensity increases strongly and abruptly, indicating the sudden appearance of nanostructured inhomogeneities in electron density. These observations confirm the earlier findings by Schmidt-Hansberg et al. who found that strong intermolecular interaction between P3HT and PCBM may inhibit the crystallization of the minority component (in their case, PCBM). ${ }^{9}$ Up to a ratio of $1: 2$, P3HT and PCBM are mixed on the molecular scale, but at a higher P3HT content, crystallites of P3HT form, creating nanoscale inhomogeneities which give a strong SAXS signal. The abrupt change is currently subject to further study and while corroborated by independent research groups ${ }^{9}$ a possible explanation in this case is that it is also a result of the short drying time ( $\sim 2$ seconds) in the coating experiment. With fast drying the crystallization threshold is likely to be defined within a very narrow range in composition space.

The most important observations we note for the ratio experiment are as follows: (1) the P3HT crystallites show the smallest coherence length at a $1: 1$ P3HT:PCBM ratio. (2) P3HT crystallizes only above a $1: 2$ ratio with PCBM, and crystallinity increases just slightly at higher ratios. (3) The lamellar spacing is consistently larger for textured $\mathrm{P} 3 \mathrm{HT}$ as compared to randomly oriented P3HT, which indicates that PCBM only interferes with the polymer chain packing in the textured crystallites. The short lamellar spacing of untextured crystallites indicates interdigitation of side chains as observed also in the thickness gradient experiment. (4) The lamellar spacing decreases with larger ratios of P3HT to PCBM showing a lessened effect of molecular interaction.

\section{Additive gradient}

The effect of solvent additives is variously described as improving phase separation, controlling donor-acceptor coherence lengths, or improving crystallinity. An example of the use of solvent additives is that of 1-chloronaphthalene $(\mathrm{CN})$ for P3HT/PCBM active layer coating from $o$-dichlorobenzene (DCB). ${ }^{15} \mathrm{CN}$ is a high boiling point solvent $\left(259^{\circ} \mathrm{C}\right)$ as compared to chlorobenzene $\left(\mathrm{CB}, 131^{\circ} \mathrm{C}\right)$ and $\mathrm{DCB}\left(181^{\circ} \mathrm{C}\right)$, and fullerenes have a much better solubility in $\mathrm{CN}$. Chen et al. concluded that these properties accounted for an improved solar cell performance because of improved phase separation and higher crystallinity of the polymer as deduced from grazing-incidence X-ray diffraction data taken along the surface normal of spin coated films. ${ }^{15}$

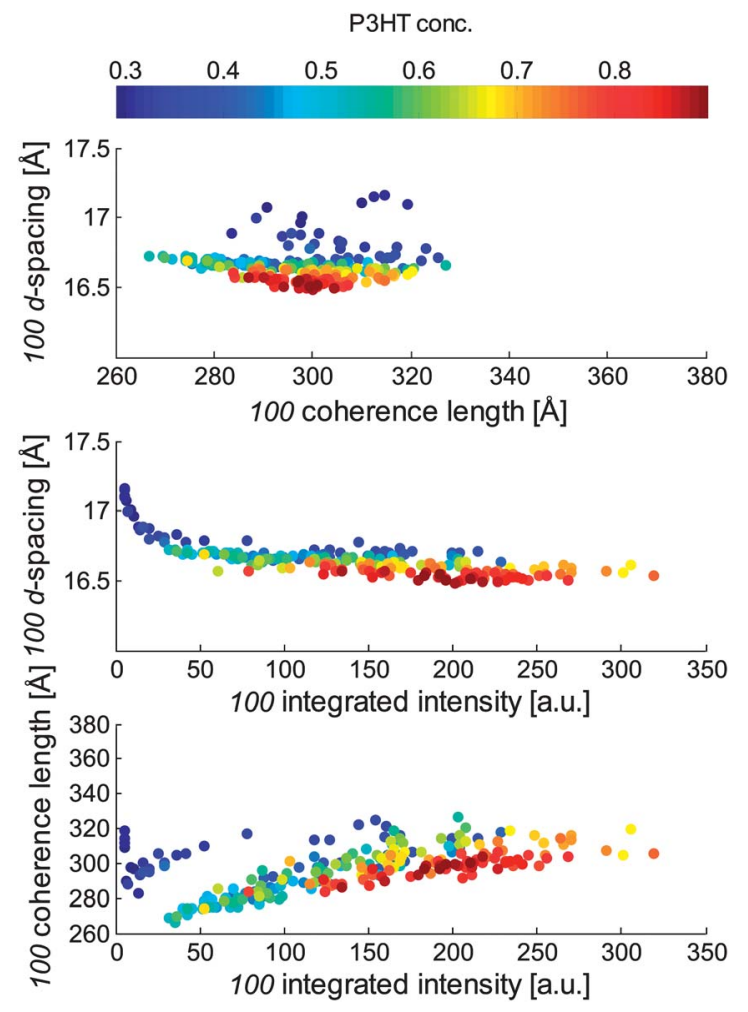

Fig. 6 Scatterplots showing relations between the parameters extracted from P3HT 100 reflections of textured crystallites for the P3HT/PCBM ratio experiment. Top panel: crystallite coherence length $v s$. $d$-spacing. Middle panel: integrated intensity $v$ s. $d$-spacing. Lower panel: integrated intensity $v s$. crystallite coherence length. The color grading corresponds to the $\mathrm{P} 3 \mathrm{HT}$ concentration $\mathrm{P} 3 \mathrm{HT} /(\mathrm{P} 3 \mathrm{HT}+\mathrm{PCBM})$, indexed as shown on the color bar at the top of the figure. 


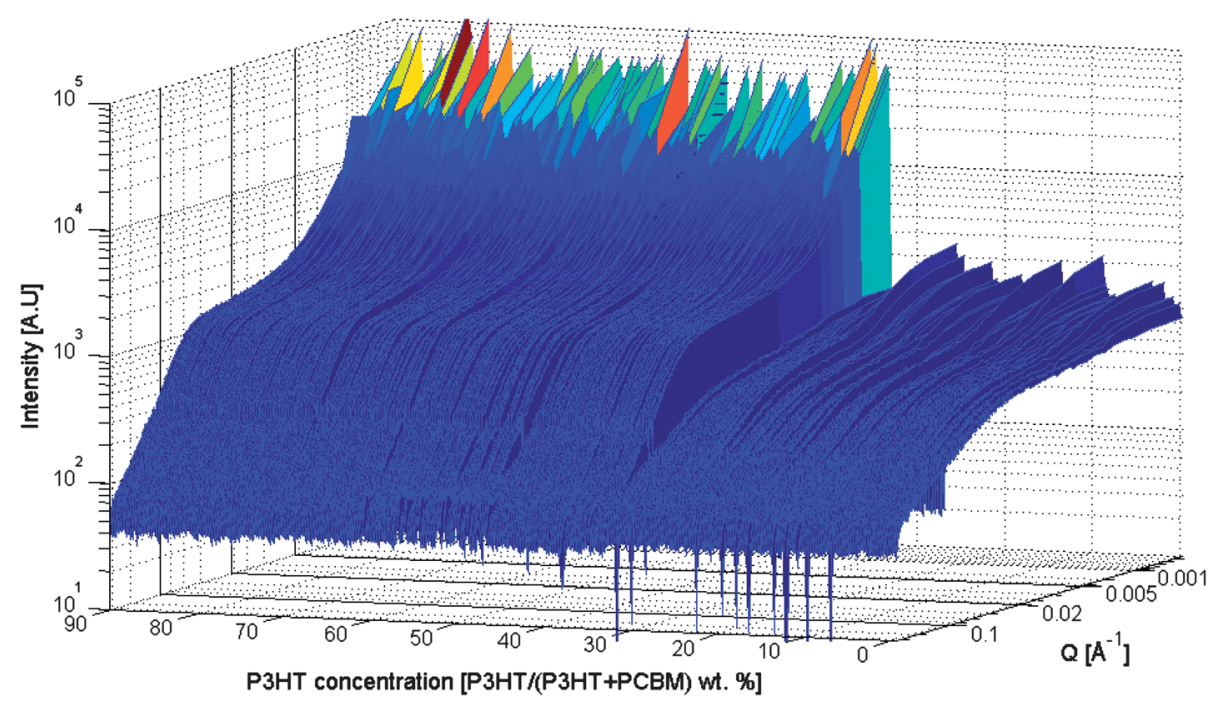

Fig. 7 GISAXS patterns from the ratio gradient experiment. The point where the scattering intensity increases dramatically corresponds to the P3HT concentration where P3HT crystallizes $(\sim 37 \mathrm{wt} \%)$.

In the present study, with the concentration of $\mathrm{CN}$ increasing from 0 to $3.5 \%$, we observe several distinct and rapid changes, corresponding to changes in polymer texture and crystallinity.

The integrated intensity of the 100 peak from the textured P3HT crystallites increases with addition of chloronaphthalene (CN), with local maxima at 1.0, 2.0 and $3.2 \mathrm{vol} \%$ (Fig. 8). The intensity of the 100 peak of untextured crystallites also shows a weak increase with $\mathrm{CN}$ concentration, albeit with a large scatter of values. The coherence length of textured crystallites is positively correlated with the scattering intensity up to about $2 \mathrm{vol} \%$ $\mathrm{CN}$ (Fig. 8, top and middle panels). The effect of $\mathrm{CN}$ on the lamellar spacing is rather striking, with a clearly significant decrease of the 100 lattice spacing of the textured crystallites and an increase of the 100 lattice spacing of untextured crystallites at 1.5 to $2.0 \mathrm{vol} \% \mathrm{CN}$. The large standard deviations for the lamellar spacings of untextured crystallites are due to a relatively high number of outliers (outside the plot range) due to poor fits of these mostly weak peaks. Nevertheless, a positive shift of about $0.1 \AA$ at 2 vol $\% \mathrm{CN}$ addition appears to be significant. The average $d$-spacing of untextured crystallites below $1.5 \mathrm{vol} \% \mathrm{CN}$ addition is 15.80(4) $\AA$ and 15.92(8) $\AA$ above 2.5 vol $\% \mathrm{CN}$ addition (standard deviations in parentheses, excluding outliers).

From the scatterplot of P3HT 100 coherence length $v$ s. lattice spacing we observe a weak negative correlation of the lamellar spacing with the coherence length, and further that the large coherence lengths appear for a $\mathrm{CN}$ concentration of $2 \mathrm{vol} \%$ and above (Fig. 9, top panel). Likewise, we observe a weak negative correlation between scattering intensity and lattice spacing with the highest intensities above 2 vol\% $\mathrm{CN}$ (Fig. 9, middle panel). In the lower panel of Fig. 9, we observe the relationship between the scattering intensity and coherence length, with a clear positive correlation up to about $2 \mathrm{vol} \% \mathrm{CN}$, above which we see a constant coherence length.

Thus, we find that the addition of a solvent with high boiling point (1) clearly improves crystallinity and (2) facilitates crystal growth, while (3) allowing time for the polymer to adopt a good molecular packing as evidenced by the short lamellar $d$-spacing in corroboration of the results obtained by Prosa et al. ${ }^{13}$ (4) The

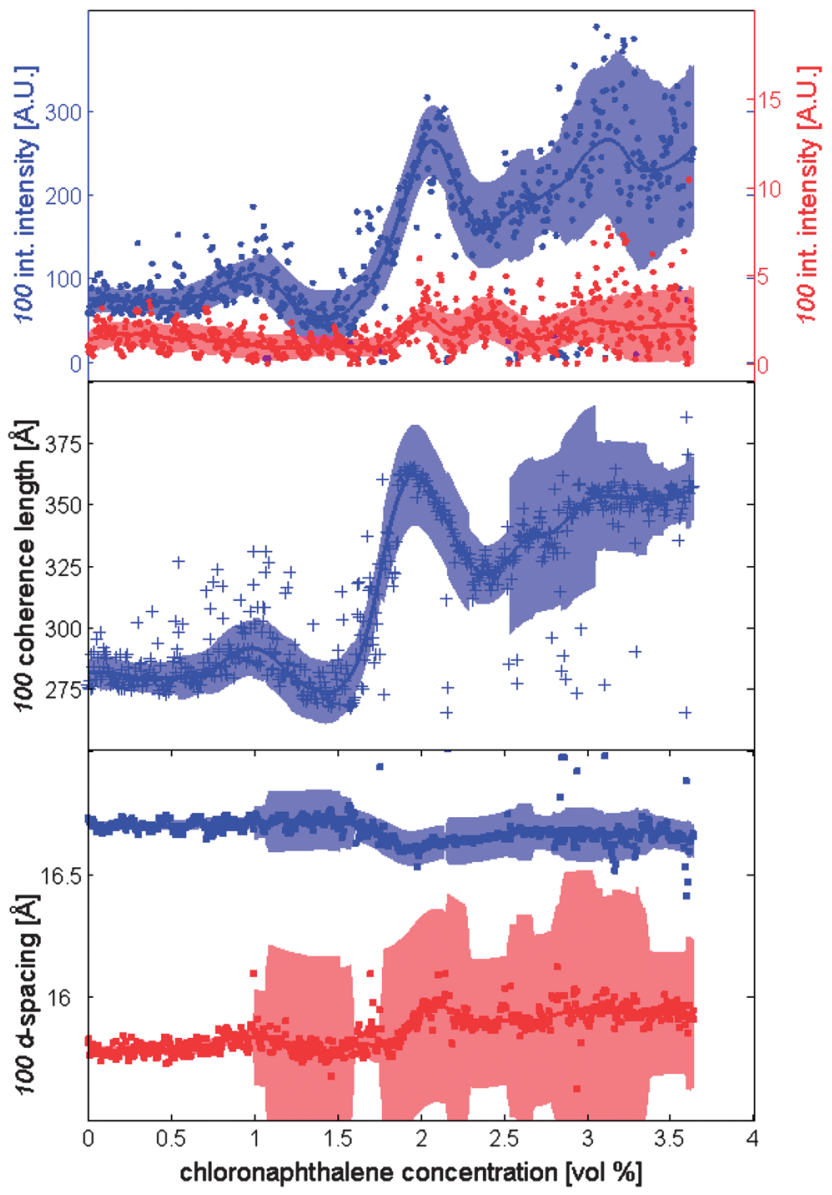

Fig. 8 Parameters of the crystalline P3HT 100 reflections shown as a function of the controlled gradient parameter, chloronaphthalene concentration. The data for the textured crystallites are shown in blue, for the untextured crystallites in red. Coherence lengths for untextured crystallites are not shown. 
chloronaphthalene conc. $\% \mathrm{v} / \mathrm{v}$
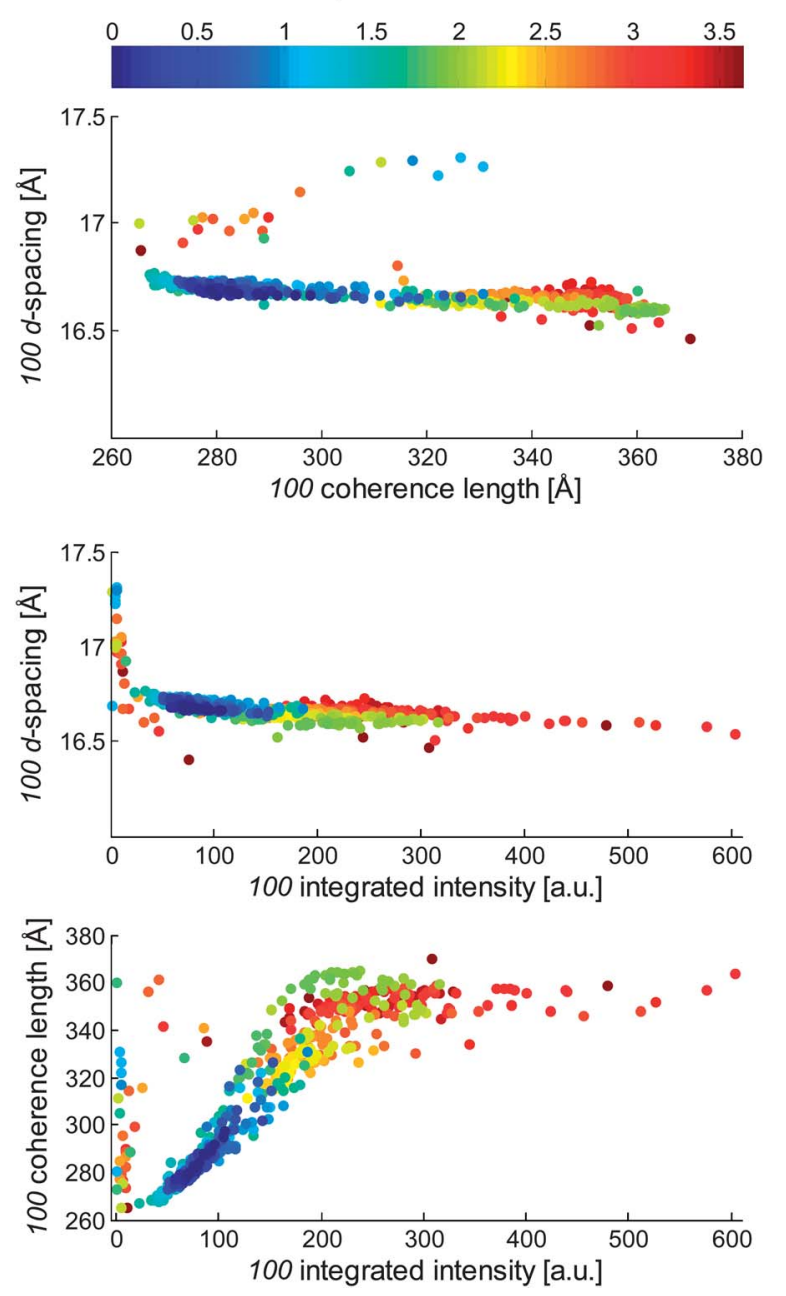

Fig. 9 Scatterplots showing relations between the parameters extracted from crystalline P3HT 100 reflections for the additive experiment. Top panel: crystallite coherence length $v s$. $d$-spacing. Middle panel: integrated intensity $v s$. $d$-spacing. Lower panel: integrated intensity $v s$. crystallite coherence length. The color grading corresponds to the $\mathrm{CN}$ concentration, indexed as shown on the color bar at the top of the figure.

strongest effect is realized at $2 \mathrm{vol} \% \mathrm{CN}$, and not much is gained by a higher percentage of additive. (5) Also at 2 vol\% $\mathrm{CN}$ and higher concentrations, the lamellar spacing of untextured crystallites increases, which may indicate that $\mathrm{CN}$ facilitates molecular interaction between PCBM and P3HT, partly interfering with side-chain ordering of the polymer.

\section{Conclusions}

We have demonstrated an efficient means of characterizing polymer crystallinity and texture of R2R coated polymer solar cell active layers as a function of a variety of controlled processing parameters. This was done by recording a high spatial density of SAXS data from the gradient coated films, varying thickness, P3HT:PCBM ratio, and $\mathrm{CN}$ additive concentration over 10 meters substrate length, using a dedicated unwinderregistration-winder unit. In this manner we were able to identify significant changes to the polymer crystalline texture as a function of active layer thickness, $\mathrm{P} 3 \mathrm{HT}$ :PCBM ratio and processing additive concentration. We find that highly textured P3HT dominates in films of up to about $270 \mathrm{~nm}$ thickness. In thicker films, we find increasing amounts of untextured P3HT crystallites, adopting a different structural form with a shorter lamellar spacing. An addition of $2 \mathrm{vol} \%$ chloronaphthalene to the processing solvent enhances the crystallization of P3HT.

This type of information can be correlated with other R2R characterization methods, including IPCE measurements, e.g. we find it significant that the minimal coherence length of P3HT coincides with the optimal 1: 1 ratio with PCBM, found in many other studies. Such correlations may facilitate fast optimization of new material combinations. We envisage that the method may be extended to online coating characterization by supplementing the roller unit with the appropriate microsyringe pumping arrangements. More detailed structural information will be available from the WAXS detector and from the GISAXS signal in the SAXS detector by performing scans at several (more shallow) X-ray incidence angles, significantly attenuating scattering from the substrate. An obvious extension of the method is for partially completed multilayer solar cell stacks which will allow a direct correlation with cell performance. Microcrystalline, inorganic materials such as the transparent conducting oxide electrode and oxide blocking layers will not have substantial scattering in the small angle regime. Finally, a subject for further study using GISAXS is crystallization phenomena as a function of drying kinetics and additives.

\section{Experimental}

\section{Materials}

Poly-3-hexylthiophene with a molecular weight of $40 \mathrm{kDa}$ and regioregularity of $96 \%$ was obtained from BASF (Sepiolid P200), PCBM was obtained from Solenne BV (99\%), chlorobenzene was of analytical grade (Aldrich, purity 99\%), and chloronaphthalene was of reagent grade (Aldrich, 97\%). The substrate was polyethylene terephthalate (PET) with a thickness of 130 micron and a web width of $305 \mathrm{~mm}$ (Dupont-Teijin, Melinex ST506). Rollto-roll coating: the method followed that described in the literature. ${ }^{2}$ The foil was labeled using an ink jet barcode printer with a unique label for each of the 6 stripes on the foil. Both a machinereadable barcode and a human-readable alphanumeric text string were written every $10 \mathrm{~cm}$ along the foil. The foil was labeled with 6 sets of 5-digit barcodes with the first digit describing the experiment number and the last 4 digits being incremented for every $10 \mathrm{~cm}$. After coating of the experiments the foil was sliced using a roll-to-roll cutting machine into rolls that were $30 \mathrm{~mm}$ wide containing only the barcode and the $13 \mathrm{~mm}$ wide coated stripe for X-ray testing.

Three different types of coating experiments were carried out in duplicate: ratio, thickness and additive experiments. For coating the web speed was $2 \mathrm{~m} \mathrm{~min}^{-1}$ with an oven temperature of $60{ }^{\circ} \mathrm{C}(2 \mathrm{~m}$ oven length). After experiment 1 had been carried out the foil was rewound and the same foil was passed through the machine while carrying out the next experiment. After the last coating experiment the foil was passed through an oven at $140{ }^{\circ} \mathrm{C}$ ( $2 \mathrm{~m}$ oven length) to anneal all stripes identically and to simulate conditions typically employed during roll-to-roll manufacture. ${ }^{16-21}$ The coating setup employs a mini slot-die coating head 
and two piston pumps with a flow resolution of $10 \mu \mathrm{L}$. The output from each pump is combined in a mixing tee and fed to the mini slot-die coating head. The coated width was $13 \mathrm{~mm}$ and the flow was fixed at $250 \mu \mathrm{L} \mathrm{min}{ }^{-1}$. In the ratio experiments, pump A contained a chlorobenzene solution of P3HT $(20 \mathrm{mg}$ $\left.\mathrm{mL}^{-1}\right)$ and pump B PCBM (20 mg mL $\left.{ }^{-1}\right)$ in chlorobenzene. In the thickness experiment, pump A contained P3HT (20 mg $\mathrm{mL}^{-1}$ ) and PCBM (20 $\mathrm{mg} \mathrm{mL}^{-1}$ ) in chlorobenzene while pump B contained pure chlorobenzene. The wet film thickness is $10 \mu \mathrm{m}$ corresponding to a starting dry thickness of $400 \mathrm{~nm}$ assuming a dry film density of $1.1 \mathrm{~g} \mathrm{~cm}^{-3}$ ( $c f$. similar experiments). ${ }^{2}$ In the additive experiment, pump A contained P3HT $\left(20 \mathrm{mg} \mathrm{mL}^{-1}\right)$ and PCBM $\left(20 \mathrm{mg} \mathrm{mL}^{-1}\right)$ in chlorobenzene while pump B contained P3HT (20 mg mL $\left.{ }^{-1}\right)$ and PCBM (20 $\left.\mathrm{mg} \mathrm{mL}^{-1}\right)$ in chlorobenzene with 1-chloronaphthalene $(5 \% \mathrm{v} / \mathrm{v})$. All solutions were stirred at $70{ }^{\circ} \mathrm{C}$ and immediately filtered ( 0.45 micro Teflon filter) before the coating experiment. In the experiment the coating was started while constantly pumping with either pump A or pump B and once the coating had been established as stable, the gradient mixing of pump A and pump B was commenced and continued over a length of $10 \mathrm{~m}$ (100 barcodes) upon completion of the gradient the coating was continued with constantly pumping pump B or pump A. After the X-ray experiments had been carried out a $4 \mathrm{~cm}$ piece of foil was cut every 1 meter and the material dissolved in a constant volume of chloroform $(4 \mathrm{~mL})$ that was subsequently analyzed using ultraviolet-visible spectroscopy (UV-vis). In this manner the start and end points of the gradient experiments were accurately confirmed (see also ESI $\uparrow$ available online).

\section{Roll-to-roll unwinder-registration-winder unit}

The device shown in Fig. 10 consists of an unwinder and winder roll, handling up to $15 \mathrm{~m}$ of coated PET substrate foil, $130 \mu \mathrm{m}$ thick. The foil is held tight to the central aluminum cylinder that serves as the X-ray measuring position. A barcode reader scans the foil continuously, registering the module number printed on

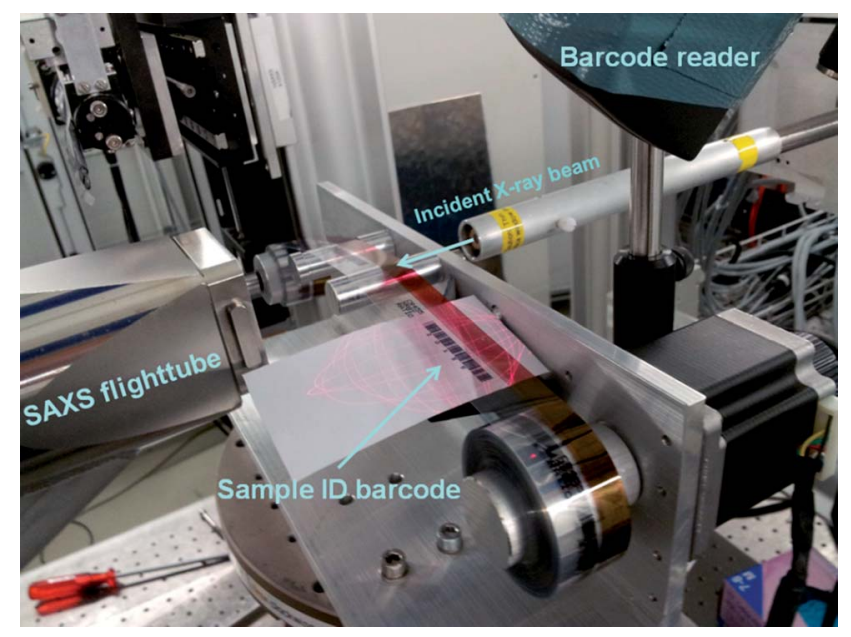

Fig. 10 The R2R unit in action at the cSAXS beam line, mounted on the sample stage hexapod. The beam flight tube exit is seen on the right and the SAXS flight tube entry on the left. On the substrate foil, the red active layer film can be seen as well as the bar code. The scanning pattern of the bar code reader laser can just be made out over the bar code. the substrate, thus keeping track of the position along the gradient experiment. The recorded module numbers are stored in the header of the X-ray data files. A stepper motor advances the foil as controlled by the beam line control software.

The entire unit was mounted on a hexapod allowing full control of the positions and tilt with respect to the X-ray beam (Fig. 10). Two modes of operation were used: first a move until a barcode was read, followed by a series of X-ray measurements at shorter spacings on the foil. This method proved somewhat unreliable, as the reading of barcodes would fail at times. We consequently changed to a mode where the foil was advanced at a constant fixed amount, followed by X-ray measurements, repeatedly, while continuously reading out barcodes for registration of the position.

\section{X-ray methods}

The experiment was carried out at the cSAXS beam line (X12SA) at the Swiss Light Source. The scattering signal was recorded on two detectors (Fig. 1). A 2 Megapixel Pilatus detector ${ }^{22}$ at $2.1 \mathrm{~m}$ from the sample behind an evacuated flight tube for measuring the small-angle $\mathrm{X}$-ray scattering signal at grazing incidence (GISAXS) and a 100k Pilatus detector ${ }^{22}$ positioned in the foil substrate plane covering angles from $13^{\circ}$ to $26^{\circ}$ to the direct beam for measuring the wide-angle X-ray scattering signal (GIWAXS). At each sampling position, four exposures of $0.1 \mathrm{~s}$ were acquired allowing $3 \mathrm{~ms}$ for readout after each. The X-ray energy was 11.1 $\mathrm{keV}$. No corrections were applied to the data. In principle, both Lorentz and polarization corrections apply, but as we are only considering variations relating to the 100 reflection in a narrow region of reciprocal space, this was deemed to be unnecessary. As the incidence angle was far above the critical angle for total reflection, the scattering intensities can be treated in the kinematical approximation, ${ }^{23}$ i.e. not requiring a correction for effects of refraction. ${ }^{24}$ From the experiment geometry we estimate an instrumental broadening amounting to about $10 \%$ of the 100 peak width in reciprocal $\AA$, corresponding to a negligible $1 \AA$ in real space assuming a Gaussian instrumental broadening. ${ }^{25}$

To obtain a signal from P3HT crystals oriented with lamellar stacks parallel to the substrate, we found that it was necessary to use an X-ray incidence angle relatively close to the angle fulfilling the Bragg condition for the 100 reflection $\left(\theta=1.92^{\circ}\right)$. The lowest incidence angle giving a satisfactory scattering intensity from the 100 reflection was found at $1.5^{\circ}$. This does however compromise the GISAXS and GIWAXS signals, because the beam penetrates into the PET substrate, giving substantial background scattering (an example of GIWAXS data is shown in the ESI $\dagger$ ). Animations showing the raw SAXS data acquired are available online.

\section{Acknowledgements}

This work was supported by the Danish National Research Foundation and the work carried out within the Danish-Chinese Center for Organic-based photovoltaic cells with morphology control. Travel support for the synchrotron experiments was provided by the Danish Center for the use of Synchrotron, X-ray and Neutron facilities 2012, DANSCATT, FNU-11109227. The authors acknowledge financial support from the European Commission under the Seventh Framework Program by means 
of the grant agreement for the Integrated Infrastructure Initiative no. 262348 European Soft Matter Infrastructure (ESMI). We gratefully acknowledge X. Donath for technical assistance with implementation of the roller setup at the cSAXS beam line and $\mathrm{T}$. Kjær for manufacturing the first roller device.

\section{References}

1 F. C. Krebs, R. Søndergaard and M. Jørgensen, Printed metal back electrodes for R2R fabricated polymer solar cells studied using the LBIC technique, Sol. Energy Mater. Sol. Cells, 2011, 95, 1348-1353.

2 J. Alstrup, M. Jørgensen, A. J. Medford and F. C. Krebs, Ultra fast and parsimonious materials screening for polymer solar cells using differentially pumped slot-die coating, ACS Appl. Mater. Interfaces, 2010, 2, 2819-2827.

3 R. Roesch, F. C. Krebs, D. M. Tanenbaum and H. Hoppe, Quality control of roll-to-roll processed polymer solar modules by complementary imaging methods, Sol. Energy Mater. Sol. Cells, 2012, 97, 176-180.

4 R. Roesch, D. M. Tanenbaum, M. Jørgensen, M. Seeland, M. Barenklau, M. Hermenau, E. Voroshazi, M. T. Lloyd, Y. Galagan, B. Zimmermann, U. Wurfel, M. Hosel, H. F. Dam, S. A. Gevorgyan, S. Kudret, W. Maes, L. Lutsen, D. Vanderzande, R. Andriessen, G. Teran-Escobar, M. Lira-Cantu, A. Rivaton, G. Y. Uzunoglu, D. Germack, B. Andreasen, M. V. Madsen, K. Norrman, H. Hoppe and F. C. Krebs, Investigation of the degradation mechanisms of a variety of organic photovoltaic devices by combination of imaging techniques-the ISOS-3 interlaboratory collaboration, Energy Environ. Sci., 2012, 5, 6521-6540.

5 L. Thrane, T. M. Jørgensen, M. Jørgensen and F. C. Krebs, Application of optical coherence tomography (OCT) as a 3dimensional imaging technique for roll-to-roll coated polymer solar cells, Sol. Energy Mater. Sol. Cells, 2012, 97, 181-185.

6 M. V. Madsen, K. O. Sylvester-Hvid, B. Dastmalchi, K. Hingerl, K. Norrman, T. Tromholt, M. Manceau, D. Angmo and F. C. Krebs, Ellipsometry as a nondestructive depth profiling tool for roll-to-roll manufactured flexible solar cells, J. Phys. Chem. C, 2011, 115, 10817-10822.

7 M. Sanyal, B. Schmidt-Hansberg, M. F. G. Klein, A. Colsmann, C. Munuera, A. Vorobiev, U. Lemmer, W. Schabel, H. Dosch and E. Barrena, In situ X-ray study of drying-temperature influence on the structural evolution of bulk-heterojunction polymer-fullerene solar cells processed by doctor-blading, Adv. Energy Mater., 2011, 1, 363-367.

8 M. Sanyal, B. Schmidt-Hansberg, M. F. G. Klein, C. Munuera, A. Vorobiev, A. Colsmann, P. Scharfer, U. Lemmer, W. Schabel, H. Dosch and E. Barrena, Effect of photovoltaic polymer/fullerene blend composition ratio on microstructure evolution during film solidification investigated in real time by X-ray diffraction, Macromolecules, 2011, 44, 3795-3800.

9 B. Schmidt-Hansberg, M. Sanyal, M. F. G. Klein, M. Pfaff, N. Schnabel, S. Jaiser, A. Vorobiev, E. Muller, A. Colsmann, P. Scharfer, D. Gerthsen, U. Lemmer, E. Barrena and W. Schabel, Moving through the phase diagram: morphology formation in solution cast polymer-fullerene blend films for organic solar cells, ACS Nano, 2011, 5, 8579-8590.

10 H. Sirringhaus, P. J. Brown, R. H. Friend, M. M. Nielsen, K. Bechgaard, B. M. W. Langeveld-Voss, A. J. H. Spiering, R. A. J. Janssen, E. W. Meijer, P. Herwig and D. M. de Leeuw,
Two-dimensional charge transport in self-organized, high-mobility conjugated polymers, Nature, 1999, 401, 685-688.

11 J. Kehres, J. W. Andreasen, F. C. Krebs, A. M. Molenbroek, I. Chorkendorff and T. Vegge, Combined in situ small- and wideangle X-ray scattering studies of $\mathrm{TiO}_{2}$ nanoparticle annealing to 1023 K, J. Appl. Crystallogr., 2010, 43, 1400-1408.

12 Y. Yoneda, Anomalous surface reflection of X rays, Phys. Rev., 1963, 131, 2010-2013.

13 T. J. Prosa, M. J. Winokur and R. D. Mccullough, Evidence of a novel side chain structure in regioregular poly(3-alkylthiophenes), Macromolecules, 1996, 29, 3654-3656.

14 N. Kayunkid, S. Uttiya and M. Brinkmann, Structural model of regioregular poly(3-hexylthiophene) obtained by electron diffraction analysis, Macromolecules, 2010, 43, 4961-4967.

15 F. C. Chen, H. C. Tseng and C. J. Ko, Solvent mixtures for improving device efficiency of polymer photovoltaic devices, Appl. Phys. Lett., 2008, 92, 103316.

16 F. C. Krebs, S. A. Gevorgyan, B. Gholamkhass, S. Holdcroft, C. Schlenker, M. E. Thompson, B. C. Thompson, D. Olson, D. S. Ginley, S. E. Shaheen, H. N. Alshareef, J. W. Murphy, W. J. Youngblood, N. C. Heston, J. R. Reynolds, S. J. Jia, D. Laird, S. M. Tuladhar, J. G. A. Dane, P. Atienzar, J. Nelson, J. M. Kroon, M. M. Wienk, R. A. J. Janssen, K. Tvingstedt, F. L. Zhang, M. Andersson, O. Inganas, M. Lira-Cantu, R. de Bettignies, S. Guillerez, T. Aernouts, D. Cheyns, L. Lutsen, B. Zimmermann, U. Wurfel, M. Niggemann, H. F. Schleiermacher, P. Liska, M. Gratzel, P. Lianos, E. A. Katz, W. Lohwasser and B. Jannon, A round robin study of flexible large-area roll-to-roll processed polymer solar cell modules, Sol. Energy Mater. Sol. Cells, 2009, 93, 1968-1977.

17 F. C. Krebs, Roll-to-roll fabrication of monolithic large-area polymer solar cells free from indium-tin-oxide, Sol. Energy Mater. Sol. Cells, 2009, 93, 1636-1641.

18 F. C. Krebs, All solution roll-to-roll processed polymer solar cells free from indium-tin-oxide and vacuum coating steps, Org. Electron., 2009, 10, 761-768.

19 F. C. Krebs, Polymer solar cell modules prepared using roll-to-roll methods: knife-over-edge coating, slot-die coating and screen printing, Sol. Energy Mater. Sol. Cells, 2009, 93, 465-475.

20 F. C. Krebs, S. A. Gevorgyan and J. Alstrup, A roll-to-roll process to flexible polymer solar cells: model studies, manufacture and operational stability studies, J. Mater. Chem., 2009, 19, 5442-5451.

21 F. C. Krebs, T. Tromholt and M. Jørgensen, Upscaling of polymer solar cell fabrication using full roll-to-roll processing, Nanoscale, 2010, 2, 873-886.

22 B. Henrich, A. Bergamaschi, C. Broennimann, R. Dinapoli, E. F. Eikenberry, I. Johnson, M. Kobas, P. Kraft, A. Mozzanica and B. Schmitt, PILATUS: a single photon counting pixel detector for X-ray applications, Nucl. Instrum. Methods Phys. Res., Sect. A, 2009, 607, 247-249.

23 P. Busch, M. Rauscher, D. M. Smilgies and D. Posselt, Papadakis grazing-incidence small-angle X-ray scattering from thin polymer films with lamellar structures - the scattering cross section in the distorted-wave born approximation, J. Appl. Crystallogr., 2006, 39, 433-442.

24 S. Lilliu, T. Agostinelli, E. Pires, M. Hampton, J. Nelson and J. E. Macdonald, Dynamics of crystallization and disorder during annealing of P3HT/PCBM bulk heterojunctions, Macromolecules, $2011,44,2725-2734$.

25 D. M. Smilgies, Geometry-independent intensity correction factors for grazing-incidence diffraction, Rev. Sci. Instrum., 2002, 73, 17061710 . 